Slavica

bruxellensia

\section{Slavica bruxellensia}

Revue polyphonique de littérature, culture et histoire

slaves

9 | 2013

Érotisme

\title{
L'image de la femme fatale dans la poésie et la peinture de la Jeune Pologne en regard des créations artistiques européennes
}

Justyna Bajda

\section{CpenEdition}

Édition électronique

URL : http://journals.openedition.org/slavica/1257

DOI : 10.4000/slavica.1257

ISSN : 2034-6395

Éditeur

Université libre de Bruxelles - ULB

Référence électronique

Justyna Bajda, «L'image de la femme fatale dans la poésie et la peinture de la Jeune Pologne en regard des créations artistiques européennes », Slavica bruxellensia [En ligne], 9 | 2013, mis en ligne le 15 avril 2013, consulté le 10 décembre 2020. URL : http://journals.openedition.org/slavica/1257 ; DOI : https://doi.org/10.4000/slavica.1257

Ce document a été généré automatiquement le 10 décembre 2020.

\section{(7) $\odot$}

Les contenus de Slavica bruxellensia sont mis à disposition selon les termes de la Licence Creative Commons Attribution - Pas d'Utilisation Commerciale - Pas de Modification 3.0 France. 


\title{
L'image de la femme fatale dans la poésie et la peinture de la Jeune Pologne en regard des créations artistiques européennes
}

\author{
Justyna Bajda
}

\section{Introduction}

1 Située entre la dernière décennie du $\mathrm{XIX}^{\mathrm{e}}$ siècle et la fin de la Première Guerre mondiale, l'époque de la Jeune Pologne est aussi unique que paradoxale dans la culture, la littérature et l'art polonais. Ce paradoxe est remarquable dans ses deux autres dénominations : le modernisme et le néo-romantisme, qui indiquent que la Jeune Pologne est perçue comme une époque complexe. D'un côté, dans toute la littérature et dans l'art polonais de la deuxième moitié du XIX ${ }^{e}$ siècle, nous observons l'énorme influence du romantisme reflétée dans l'effusion de sentiments (sans oublier l'héritage de la nation oppressée depuis presque un siècle par les trois puissances: la Prusse, l'Autriche, la Russie), de la passion longuement contenue et la soif de liberté d'expression. Aussi, en traversant l'époque du réalisme, les Polonais ont-ils ressenti de nouveau, à la fin des années 1880 , un grand besoin de se tourner vers le romantisme, vers une grande apothéose de l'individu et des idées transcendantales et mystiques. D'un autre côté, à cette époque, des représentants de la jeune génération proposent d'oublier le passé, de vivre dans le présent et d'ouvrir la Pologne à l'Europe de l'ouest, aux nouvelles tendances littéraires et artistiques. Ce sont eux qui cherchent alors à établir des contacts avec les plus importants centres culturels, et plus particulièrement avec la capitale française. Ces démarches ne sont pas restées infructueuses. Grâce à leurs efforts, la littérature et l'art polonais ont rapidement connu l'influence de plusieurs courants artistiques, tels que le naturalisme, l'impressionisme, le symbolisme et finalement l'Art nouveau. On y distingue aussi les motifs iconographiques les plus 
connus de la peinture et de la poésie européennes de la fin du XIX siècle, parmi lesquels la femme et l'amour qui se trouvaient alors au sommet de leur popularité.

2 L'image de la femme dans la littérature et l'art de cette époque est hétérogène et pleine de contradictions. Premièrement, elle est directement liée à la vie moderne de la Belle Époque : à son rythme et à sa verve. Mais le portrait de la femme reflétait - comme dans un miroir magique - toutes les angoisses et toute la lassitude de la fin du siècle. En Pologne, il faut encore joindre à cette image (déjà bien complexe) les représentations de personnages féminins immortalisés par les œuvres de l'époque du romantisme. C'est pourquoi l'image de la femme dans la peinture et dans la poésie de la Jeune Pologne est intrigante et peut éveiller une certaine curiosité.

3 Parmi les nombreux types de jeunes filles, de belles femmes ou de vieilles matrones qui sont les sujets privilégiés de la production artistique européenne et polonaise de la fin $\mathrm{du} \mathrm{XIX}^{\mathrm{e}}$ siècle, nous reconnaissons celui de la femme fatale: il s'agit du type le plus controversable mais aussi du plus fascinant. Cependant, comme le constate Mario Praz, ce n'est pas la seule imagination des artistes et des poètes qui en a fourni les images les plus remarquables pendant des siècles. Une présence constante de la femme fatale dans la mythologie et dans la littérature européennes est motivée par des exemples puisés dans la réalité :

(...) La mythologie et la littérature reflètent, sur le plan de l'imagination, différents aspects de la réalité - et la réalité a toujours fourni des exemples plus ou moins bons d'une féminité despotique et cruelle. ${ }^{1}$

4 Dans la littérature de la première moitié du XIX ${ }^{e}$ siècle, Praz distingue entre autres différents types de femmes fatales tels que la femme-diable, la femme-coquette, la femme-démon, et parmi les héroïnes historiques et légendaires : la reine de Saba, Cléopâtre, Messaline, Hérodiade ou La Belle Dame Sans Merci. ${ }^{2}$ Bien que toutes ces images de femmes fatales semblent avant tout être déterminées par leur caractère énigmatique et leur attitude provocante et séduisante envers les hommes ${ }^{3}$, d'autres éléments entrent en jeu dans la constitution de ce personnage. D'après nous, deux facteurs capitaux déterminent les différents types de représentations des femmes fatales dans la littérature et dans l'art. Le premier est le facteur chronologique et social - il concerne le contexte historique et socioculturel dans lequel les images de ces femmes ont été forgées. Le deuxième touche au style de l'époque (à la fin du XIX ${ }^{\mathrm{e}}$ siècle, il s'agit surtout de l'Art nouveau) et à l'iconographie qui se manifeste par les thèmes et les motifs les plus courants.

5 Dans notre article, nous nous concentrerons sur trois représentations distinctes de la femme fatale "à la polonaise", qui correspondent à des figures célèbres de la littérature et de l'art européens de la fin du XIX ${ }^{e}$ siècle. Les types proposés sont également les plus intéressants au niveau de la production littéraire et artistique et les plus fréquents dans la poésie et l'art polonais.

6 Tout d'abord, nous présenterons les figures de femmes fatales contemporaines, c'est-àdire des figures appartenant entièrement à la réalité de l'époque de la Jeune Pologne. Les artistes essayaient alors de créer des portraits de femmes vivant d'un côté en société - en particulier dans la société bourgeoise ou petite-bourgeoise, patriarcale et intolérante - mais sachant de l'autre rester mystérieuses et énigmatiques. Ce sont ces femmes-là qui ont rompu les traditions consacrées par des générations et qui ont défié toutes les convenances de l'époque. En conséquence, l'image de la femme moderne a pu devenir parfois égale à l'image de la femme fatale. Cette façon de conceptualiser la 
femme dans la littérature polonaise s'explique habituellement par la perspective sociale, éthique ou par la tradition à laquelle elle se réfère. Le caractère diabolique des femmes était lié à une certaine projection misogyne(comme chez August Strindberg, Lev Tolstoj, Otto Weininger, Friedrich Nietzsche). À ce propos, Wojciech Gutowski écrit :

(...) La haine pour le beau sexe est considérée comme une forme de défense contre le danger que les hommes (jusqu'alors, les créateurs incontestables de la culture) ressentent devant l'offensive des femmes émancipées, qui rivalisent avec succès avec eux dans le domaine professionnel et qui sont de plus en plus actives dans les relations sexuelles. ${ }^{4}$

7 Comme deuxième type de femmes fatales nous proposerons ensuite de distinguer un ensemble de figures bibliques. Ces héroïnes apparaissent aussi bien dans la peinture et la littérature de la Jeune Pologne que dans celles de toute la Jeune Europe. Dans l'art polonais, nous reconnaissons plusieurs personnages de femmes de l'Ancien et du Nouveau Testament, avecentre autres : Lilith, Ève, Judith, Hérodiade, Salomé et Marie Madeleine. Les héroïnes bibliques, vues par les artistes et les poètes dans une perspective culturelle et esthétique, deviennent les femmes fatales les plus célèbres de l'époque mais, en même temps, peuvent être considérées comme représentant des êtres définitivement éloignés de toute vision de fatalité et de féminité séduisante.

Dans un dernier temps, nous nous intéresserons à des types de femmes plus rares dans l'art et dans la littérature de la Jeune Pologne mais qui sont, dans l'opinion commune, les figures les plus spectaculaires : il s'agit des personnages historiques, mythiques et surtout fantastiques. Il semble que, parmi ces trois manifestations artistiques, le type de la femme-hybride, représenté dans des œuvres d'artistes bien connus à l'époque, soit le plus riche et le plus intéressant.

9 La femme fatale «à la polonaise » est en même temps admirée et méprisée. Cette ambivalence de la femme, qui n'est plus uniquement un être parfait, surnaturel et éternel (comme elle l'était dans l'imaginaire national polonais encore à l'époque du romantisme), a été immortalisée par de nombreux peintres et écrivains de la fin du XIX ${ }^{\mathrm{e}}$ siècle.

10 L'objectif principal de cet article est de présenter aux lecteurs francophones différentes images des femmes fatales issues de la création littéraire et artistique de la Jeune Pologne, en montrant les caractéristiques qu'elles partagent avec les créations artistiques européennes. Dans ce but nous analyserons les trois types de femmes fatales distingués ci-dessus, tout en nous appuyant sur quelques exemples essentiels. Ces exemples sont tirés d'une part d'œuvres poétiques et romanesques et d'autre part de productions picturales. Nous tenons à préciser que l'aspect que nous privilégierons dans notre analyse des œuvres littéraires sera l'aspect iconographique.

\section{La femme contemporaine}

11 Le premier type d'image de femme qui doit être distingué est celui de la femme contemporaine - une femme vivant dans la réalité de son époque. Sa représentation subit des transformations multiples dans l'art polonais du XIX ${ }^{\text {e }}$ siècle: depuis les portraits romantiques, en passant par les représentations réalistes, la grande peinture académique et les compositions plus intimes (et beaucoup plus douces), appelées peinture de salon ${ }^{5}$, jusqu'aux images expressionnistes de Stanisław Ignacy Witkiewicz 
dans les années $1920^{6}$. Entre ces exemples extrêmement différents se trouvent encore d'autres types de portraits, comme les silhouettes de femmes délicates et très sensuelles réalisées par Teodor Axentowicz. Le pastel Wiosna (Le printemps, vers 1900) présente l'image d'une jeune fille nue au torse d'albâtre. Son corps idéal exprime toute sa sensualité : ses cheveux longs et roux, son cou élancé, ses mains délicates, ses yeux grands et mi-clos et enfin ses lèvres épaisses. La fille porte dans ses mains un miroir et une fleur de tulipe jaune, pouvant symboliser les sens humains que sont la vue et l'odorat. Ces attributs renvoient le spectateur aux images allégoriques typiques du $\mathrm{XVII}^{\mathrm{e}}$ et XVIII ${ }^{\mathrm{e}}$ siècles, réunies et publiées par Cesare Ripa dans sa célèbre Iconologie en $1593^{7}$. Le peintre a créé beaucoup de figures semblables mais nous trouvons aussi chez lui des images qui cachent, sous leur sensualité, un mystère, une sorte de fatalité. Elles ne sont jamais définitivement achevées et restent ouvertes à plusieurs interprétations. ${ }^{8}$

La femme fatale de l'époque est toujours entourée d'une énigme, de secrets, de l'intimité de sa vie privée. Elle connaît bien la mentalité des hommes et elle sait les provoquer, les séduire, les tenter avec sa sensualité. En même temps, elle demeure dans l'ombre de la grande ville, elle préfère l'obscurité, se cache dans des cafés ou dans des cabarets. Une telle image de la femme est peu représentée dans la peinture polonaise de la fin du XIX ${ }^{e}$ siècle, mais elle apparaît par exemple sur la toile de Leon Kaufmann (Kamir) intitulée Ćma nocna (Papillon de nuit, 1899)9 , qui présente une femme fatale moderne. Dans ce tableau, une obscurité opaque masque toute la silhouette féminine. Nous n'apercevons que le visage pâle d'une jeune fille, éclairé par la faible lumière d'un réverbère, et un énorme chapeau au voile noir qui ressemble aux ailes d'un papillon de nuit. Elżbieta Charazińska décrit ce type d'image de la façon suivante :

La femme fatale de la Jeune Pologne était une héroïne des paysages urbains nocturnes. Enveloppée dans du noir, regardant d'une manière séduisante du dessous de son chapeau aux ailes larges, elle constituait une des figures incontournables de la ville. Pour les artistes de la fin du siècle, habillée ou nue, elle était un symbole de la puissance des sentiments et de la passion capable de détruire chaque homme. ${ }^{10}$

13 Il est indispensable d'évoquer ici un autre tableau, connu de tous les Polonais, qui est devenu un vrai symbole de la fatalité et de l'érotisme de l'époque. Il s'agit d'une œuvre de Władysław Podkowiński, Szał uniesień (La Folie, 1894), qui représente le nu d'une femme aux cheveux roux, enfourchant un cheval à cru. Cabré au bord d'un gouffre, l'animal prend l'allure d'une bête apocalyptique: il a la crinière noire flottante, la bouche ouverte, la langue tirée, l'œil grand et rouge. La femme l'étreint en passant les bras autour de son cou. Les frontières entre ces deux êtres se confondent, ils ne font qu'un. Dès le vernissage à Varsovie en mars 1894, le tableau suscita un grand intérêt mais il inspira en même temps de la répulsion et souleva de nombreuses objections. Il fit littéralement sensation: les visiteurs et les critiques n'y voyaient que l'érotisme provocateur et la fatalité du corps de la femme. On peut supposer que la femme qui servit de modèle était Ewa Kotarbińska. Célèbre dans les milieux varsoviens, Podkowiński avait fait sa connaissance pendant ses vacances dans le château de Wincentyna Karska près de Varsovie. Le grand scandale, qui éclata pendant le vernissage, entraîna la destruction du tableau par l'auteur juste avant la fin de l'exposition (le 24 avril) ${ }^{11}$.

14 La Folie a été décrite dans un drame de Jan August Kisielewski ${ }^{12}$ et dans quelques poèmes parmi lesquels nous citerons ceux de Zofia Gordziałkowska et de Maria Komornick $a^{13}$. Ces poèmes qui présentent une grande force d'érotisme sont autant d' 
ekphraseis qui influencent les lecteurs grâce à des descriptions vives et pittoresques. Le poème le plus coloré est celui de Gordziałkowska où tous les motifs iconographiques de la peinture sont cités. Le leitmotiv du texte est un cheval dont la sensualité s'exprime par une figure dynamique ainsi que des détails anatomiques. Parmi les phrases le décrivant, citons : «Il court... à la crinière flottante - le feu s'exhale de ses naseaux / Les yeux affolés, injectés de sang, flambent. $»^{14}$ Une femme se manifeste quelques lignes plus bas : totalement nue, auréolée de ses cheveux épars, "elle boit avidement un courant d'air avec sa poitrine blanche / (...) / (...) - et dans ses yeux et sur son visage blanc / Le sentiment de satisfaction se lie à la torture $»^{15}$. Gordziałkowska ne montre que les attributs de la femme fatale en construisant son portrait au moyen des valeurs érotiques du corps. Les émotions et les sentiments sont explicités eux aussi par le truchement d'une représentation physique plongée dans un paysage infernal. Toute cette image poétique, de mauvaise qualité sur le plan artistique mais très emblématique pour la sensibilité des poètes mineurs de la Jeune Pologne, est empreinte de mots exaltés et passionnés. À la fin même du poème, la femme poète prend soin de ne pas mettre un terme à ses divagations poétiques, en posant une question rhétorique : « [Le cheval] va$\mathrm{t}$-il tomber en arrière ou va-t-il sauter dans le gouffre ?! $»^{16}$

Passons maintenant à un poème différent, celui de Komornicka, une femme fascinante en tant qu'artiste mais tragique dans son destin d'humain ${ }^{17}$. Son poème est entièrement consacré à la folie d'amour qui ne peut conduire qu'à la mort. C'est un texte tout à fait moderne et - ce qui n'était pas fréquent à l'époque pour les femmes auteurs - écrit de la perspective d'un homme! Komornicka retranscrit l'atmosphère pesante de l'amour érotique, qui concentre les corps, le sens du toucher, les caresses et la douleur. L'amour est un sentiment fatal et ambivalent pour elle : la douceur du bonheur et le goût amer du poison. ${ }^{18}$

Pour conclure notre commentaire de la peinture de Podkowiński, nous nous permettrons d'alléguer les mots de Kazimierz Przerwa-Tetmajer, un des plus grands poètes de la Jeune Pologne. Après avoir admiré ce tableau lors du vernissage, il a écrit à son sujet un article où il n'était question que de la fatalité, de la sensualité et du désir de la femme moderne :

Elle flambe - ce coursier fou, c'est la folie de ses désirs, c'est le volcan de ses passions, vomissant des laves et du vent enflammé, c'est la fureur de son sang, de son imagination, de son corps. ${ }^{19}$

Il est intéressant de noter que Tetmajer, considéré par les lecteurs (et surtout par les lectrices) et les critiques littéraires de la Jeune Pologne comme l'écrivain le plus sensuel de l'époque, n'a laissé parmi ses œuvres aucune interprétation poétique du tableau de Podkowiński. Mais il faut admettre que le portrait de la femme fatale de la fin du XIX ${ }^{\mathrm{e}}$ siècle n'aurait été complet sans certains poèmes de Tetmajer, cités jusqu'aujourd'hui dans chaque anthologie de poésie de la Jeune Pologne. Il s'agit notamment de quelques poèmes érotiques (avec par exemple les titres : «Moi, quand j'approche ma bouche de ta bouche... »; "J'aime, quand une femme... $»^{20}$ ) et d'ekphraseis décrivant des peintures et des statuaires de femmes, comme Léda et le Cygne ${ }^{21}$ (le tableau de Michel-Ange), et $D_{a n a e^{2}}$ (la peinture de Titien).

Les ekphraseis de Tetmajer sont avant tout des poèmes érotiques bien cachés sous le masque des œuvres de la Renaissance et de la mythologie. Les femmes décrites par le poète se trouvent dans des situations clairement sensuelles. La belle Léda, aux proportions idéales d'une sculpture de Michel-Ange, «s'évanouit de volupté entre les 
ailes du cygne ». Pour le poète, elle est le plus grand symbole de la sensualité. Dans l'article déjà cité, Tetmajer compare également deux images de femme, celle de Podkowiński et celle de Michel-Ange :

Il y a le chef-d'œuvre Léda et le Cygne de Michel-Ange. Je ne sais pas si on peut exprimer plus génialement une adoration et une sujétion à la volupté, le calme d'une passion assouvie : Léda s'évanouit, inerte, dans une sorte d'ivresse extatique s'approchant du nirvana - - - ce n'est pas comme la femme de Podkowiński. Celle-là désire, elle désire de tout son être, de chacun de ses nerfs, de chaque goutte de son sang. ${ }^{23}$ dans la littérature et l'art, en Pologne comme dans le reste de l'Europe. En respectant l'ordre chronologique, nous citerons d'abord des femmes de l'Ancien Testament, à commencer par Lilith, personnage légendaire, dont le nom paraît dans le Livre d'Isaac ( 34 :14). Antoni Lange, un poète polonais de l'époque, a écrit le sonnet «Lilith ${ }^{28}$ qui - 
sous un masque biblique - présente une image typique de la femme fatale moderne: très ambivalente, elle devient pour l'homme à la fois une bénédiction et une malédiction. En tant que première femme fatale dans l'histoire du genre humain, Lilith est également présente sur les toiles des artistes européens du XIX siècle : la femme, d'une beauté accomplie, au corps idéal et aux cheveux roux ${ }^{29}$ est parfois enlacée par un serpent $^{30}$.

Il en est de même pour Ève, sombre héroïne d'une fameuse peinture de Franz von Stuck intitulée Le péché (1893), sur laquelle nous ne voyons que son visage, son torse nu, ainsi qu'un grand reptile autour de son corps. La toile est devenue très rapidement célèbre en Europe, y compris en Pologne. La poétesse polonaise Maria Konopnicka a écrit un article entièrement consacré à ce tableau scandaleux, après avoir vu une exposition à Vienne. Nous y trouvons cette phrase emphatique sur la vision d'Ève qui correspond aux images fatales :

Le visage de la femme - pâle-fougueux, plutôt mûr que printanier, le corps - mince,

(...) les yeux - concupiscents, la bouche - pleine du goût d'une douceur secrète, au-

dessus de son sein droit - une tête vert doré de serpent, plongée dans l'éclat et dans

les couleurs des pierres précieuses. ${ }^{31}$

Judith est une autre héroïne de l'Ancien Testament qui a fait fureur à la Belle Époque. ${ }^{32}$ La jolie veuve qui a sauvé sa ville, Béthulie, assiégée par l'armée de Nabuchodonosor roi d'Assyrie, était sujette à discussion, souvent comparée à un personnage du Nouveau Testament - Salomé..$^{33}$ À la fin du XIX ${ }^{\mathrm{e}}$ siècle, les portraits des deux femmes étaient présentés de manière similaire. Dans la plupart des cas, nous observons une jeune et belle femme qui tient à la main un attribut caractéristique : une tête masculine coupée. Les artistes semblaient oublier que les causes des décapitations d'Holopherne et de saint Jean-Baptiste étaient totalement différentes, et ils présentaient aux spectateurs la même personnification de la sensualité féminine. Dans la littérature européenne, les descriptions de Salomé sont particulièrement nombreuses. Elle est présentée comme un être provoquant par sa corporalité et sa volupté, séduisant des hommes au moyen de la danse. La fille d'Hérodiade est devenue entre autres l'héroïne d'un poème de Stéphane Mallarmé («Hérodiade», 1869), d'une nouvelle de Gustave Flaubert (Hérodiade, 1877) ou encore d'une tragédie en un acte d'Oscar Wilde (Salomé, 1892). En 1884, Joris-Karl Huysmans écrit le roman À rebours où il insère quelques descriptions des tableaux de Gustave Moreau, consacrés à la danse de Salomé. Plusieurs esquisses et d'autres peintures à l'huile du symboliste français ont sans doute contribué à l'énorme popularité de la princesse biblique à la fin du siècle et sont devenues une source d'inspiration pour des réinterprétations littéraires et poétiques du thème. Leur valeur émotionnelle est surtout appréciée par le personnage principal du roman de Huysmans : le duc Jean des Esseintes. ${ }^{34}$ En ce qui concerne la Pologne, c'est Zuzanna Rabska, poétesse méconnue, qui s'est penchée sur ces tableaux. En tant que grande admiratrice d'art, elle a composé, à la fin de l'époque, un petit poème ekphrastique d'après une œuvre de Moreau (L'Apparition, 1876). Le sonnet exprime son admiration pour l'esthétisme et la poétique de l'époque et - dans une perspective contemporaine se caractérise par "une surabondance des mots». Nous y trouvons par exemple des phrases multipliant les adjectifs qualificatifs, pleines d'une emphase typique pour la poésie de la Jeune Pologne, telles que: "Soudain, elle s'est figée dans un spasme de folie flamboyante "; « Son jeune corps brûle d'un désir purpurin »; « Ses prunelles sont un seul cri de désir $\aleph^{35}$. Sous cette profusion d'expressions banales, de formes sans grande originalité, de descriptions prosaïques, nous devinons sous quels traits Salomé - 
femme fatale et décadente - était identifiée à l'époque. Mais il faut reconnaître que les descriptions de Judith étaient fortement similaires! Pour comprendre ce phénomène artistique, il suffit de rappeler deux œuvres de Gustave Klimt : Judith I (1901) et Judith II (1909). Sur un fond de mosaïques dorées, l'artiste dessine les portraits de femmes considérées comme la quintessence de la fatalité de la Belle Époque. Cette quintessence provient surtout de l'ambivalence de l'image : une femme belle et cruelle, un être à la douceur angélique qui apporte le Mal. C'est peut-être la raison pour laquelle Klimt a proposé deux titres pour son tableau de 1909 : Judith et... Salomé.

Parmi les autres artistes qui s'intéressaient à l'époque à ce motif, nous pouvons ajouter encore le peintre polonais Kazimierz Wasilkowski et son portrait de Salomé datant de 1912. L'artiste a peint une femme calme mais consciente de sa valeur et de sa sensualité. Il a dévoilé sa poitrine et a décoré son décolleté de bijoux. Ses hanches sont entourées par une riche ceinture en cuir, avec au centre une grande boucle d'argent, sur laquelle nous découvrons les contours d'un serpent sinistre.

L'analyse de l'image de Salomé ne serait pas complète si nous négligions la présence des éléments nécrophiles. Ce motif est devenu célèbre grâce aux dessins d'Aubrey Beardsley (1893), réalisés pour un drame connu de Wilde, dans lequel nous reconnaissons ce personnage plein d'érotisme et de volupté. Mais la peinture de Lovis Corinth (1900) n'en est pas exempte non plus. Elle pourrait illustrer ce monologue de Salomé, déclamé devant la tête coupée d'Iokanaan (saint Jean-Baptiste), apportée par les soldats sur un bouclier d'argent :

Ah! tu n'as pas voulu me laisser baiser ta bouche, Iokanaan. Eh bien ! je la baiserai maintenant. Je la mordrai avec mes dents comme on mord un fruit mûr. Oui, je baiserai ta bouche, Iokanaan. Je te l'ai dit, n'est-ce pas? je te l'ai dit? Eh bien! je la baiserai maintenant.... ${ }^{36}$

Et quelques pages plus loin, l'acte nécrophile est accompli :

Ah ! j'ai baisé ta bouche, Iokanaan, j'ai baisé ta bouche. Il y avait une âcre saveur sur tes lèvres. Était-ce la saveur du sang ?... Mais peut-être, est-ce la saveur de l'amour. On dit que l'amour a une âcre saveur... Mais, qu'importe? Qu'importe? J'ai baisé ta bouche, Iokannan, j'ai baisé ta bouche. ${ }^{37}$

Dans la littérature polonaise, nous trouvons des passages semblables. Gutowski constate que justement dans les œuvres de la Jeune Pologne, nous trouvons des exemples notables de cette "symphonie du sang $»^{38}$. C'est surtout Salomé qui est présente chez Jan Kasprowicz, un des plus grands poètes de la Jeune Pologne. D'après nous, dans l'hymne «Salomé » (1899) ${ }^{39}$, Kasprowicz a formé une image de la princesse biblique qui s'inscrivait entièrement dans le paradigme de la femme fatale, décrite par Praz comme un être cruel, ayant des inclinations sadomasochistes, mais apparaissant parallèlement comme un être extatique et coquet. Les attributs de sa sensualité restent toujours ses cheveux roux ou noirs, ses grands yeux et son visage pâle. ${ }^{40}$ Nous pouvons conclure que ce portrait convient parfaitement à la Salomé de Kasprowicz : une femme belle et démoniaque, agissant sur tous les sens des hommes, éveillant le désir, mais aussi une femme raffinée, orientée vers la sensation de volupté, qui provoque la douleur. C'est quelqu'un qui ne suit que son penchant pour le pouvoir et le Mal. Son aspect physique est conforme au canon de la femme que nous retrouvons dans l'Art nouveau : son visage est très clair, elle a les cheveux roux (et même : « cuivrés ») et les yeux brillants. dans d'autres ouvrages de la Jeune Pologne. Elle est d'ailleurs mentionnée comme 
l'héroïne d'un sonnet de Kazimiera Zawistowska intitulé " Hérodiade ». Il est difficile d'affirmer à quelle femme biblique nous pourrions attribuer ce monologue exalté qui commence par les phrases suivantes : «Connais-tu la volupté ? / Le murmure des fleurs pâles ne t'émouvra-t-il pas dans ces nuits étouffantes? / Viens... je vais dénatter mes cheveux noirs pour toi / Je vais entrer par le serpent des désirs dans ton âme $»^{41}$. Nous pensons que tous les traits évoqués dans le texte peuvent caractériser aussi bien Salomé que sa mère, l'instigatrice du meurtre du prophète biblique.

Une autre femme du Nouveau Testament, Marie Madeleine, se distingue aussi dans plusieurs œuvres littéraires et artistiques, bien qu'elle fût moins populaire à l'époque que les femmes précédentes. C'est autour de ce personnage que se produit l'intrigue du roman de Gustaw Daniłowski Marie Madeleine ${ }^{42}$. Un critique polonais, Dariusz Trześniowski constate que l'auteur a doté son héroïne de traits propres à un être fatal :

[Elle est] montrée (de même que la Salomé de Kasprowicz) comme un idéal de beauté de l'Art nouveau, c'est " une femme envoûtée par Éros », une femme qui a un tempérament violent, aux désirs inassouvis, cherchant une satisfaction androgyne. C'est pourquoi elle devient la Dame aux Camélias de Jérusalem, elle a de nombreux amants mais aussi des expériences lesbiennes; et elle est même souvent - comme Salomé - un vampire qui boit du sang (le sang d'autrui ou son propre sang, coulant des stigmates). ${ }^{43}$

31 De même, nous rencontrons souvent cette femme dans la poésie polonaise, mais elle n'est pas toujours caractérisée comme un être fatal. Nous pouvons supposer que les poètes voulaient distinguer quelques personnages bibliques comme Marie - la pécheresse, Marie de Magdala (Marie la Magdaléenne) et Marie de Béthanie.

Madeleine est par exemple l'héroïne du poème "Eloé et Madeleine » de Bronisława Ostrowska $(1911)^{44}$. C'est une femme à deux visages : elle est déchirée entre un idéal spirituel et un bonheur humain. Quelques années plus tard, Ostrowska a écrit un poème «Madeleine, silence de champ! ...» (1919) ${ }^{45}$, considéré comme une des dernières mais aussi une des plus belles œuvres lyriques de la Jeune Pologne, qui fait le portrait d'une femme définitivement éloignée de toutes les images de femmes fatales. Cette fois-ci c'est un personnage qui suit les règles de saint François, qui vit en pleine harmonie avec la nature et qui rencontre Jésus-Christ sur son chemin à travers un champ.

Pour conclure cette partie consacrée aux femmes bibliques, nous pouvons affirmer sans nul doute que, parmi toutes les femmes fatales bibliques, c'est Salomé qui représente la femme-type la plus importante à l'époque de la Jeune Pologne. Contrairement aux textes des Évangiles, elle est représentée comme une personne entièrement consciente de tout ce qu'elle veut. Salomé devient dans la littérature polonaise la femme la plus sensuelle et la plus dangereuse, un vrai démon détruisant les hommes qui s'approchent d'elle. En revanche, Marie Madeleine n'est pas un personnage univoque et peut être perçue comme la personnification d'un chemin menant du péché et de la fatalité à l'amour idéal.

Les deux femmes sont, d'après Trześniowski :

(...) Les grandes maîtresses, possédées par un besoin d'affection, les deux inapaisées dans leurs recherches. Mais, ce qu'il faut souligner, c'est que le but de Salomé était l'état d'hystérie émotionnelle, et même l'état de folie, tandis que Marie Madeleine a trouvé la paix, accomplie par la mort. Cependant, dans les deux cas, on a perdu la vie. ${ }^{46}$ 


\section{Les personnages historiques, mythiques et fantastiques}

Nous ne saurions oublier les personnages historiques apparaissant de temps en temps aussi bien dans la peinture que dans la littérature européenne de la fin du XIX ${ }^{\mathrm{e}}$ siècle. Toutes ces grandes maîtresses du passé, elles aussi, représentaient un type de femme fatale. Parmi les exemples notables, nous aimerions citer ceux qui proviennent du cycle poétique «Dusze» (Les Âmes) de Zawistowska, une des poétesses polonaises qui figuraient l'amour, les émotions et la fatalité dans une perspective féminine. Dans ces quelques poèmes, nous trouvons les personnages féminins les plus beaux, les plus amoureux mais aussi les plus tragiques de "l'histoire de l'amour»: Cléopâtre, Agrippine et Madame de Pompadour. Bien que toutes aient été présentées comme des femmes fatales, chacune est caractérisée d'une manière singulière, insérée dans un paysage pittoresque. Dans l'entourage le plus proche de Cléopâtre, nous ressentons surtout le grand désir de son mari Marc Antoine et celui de son amant Jules César. Ces hommes remettent leur âme entre les mains de leur maitresse et deviennent ses serviteurs. ${ }^{47}$ C'est une image bien différente de celles que nous admirons sur les toiles des peintres européens..$^{48}$ Premièrement, nous pouvons remarquer que les artistes étaient essentiellement inspirés par la beauté exceptionnelle de la reine d'Egypte ou encore par le moment de sa mort. Dans la plupart des exemples, Cléopâtre est présentée comme une femme ravissante et désirable, au moment de son premier rendez-vous avec l'un des deux hommes de sa vie. Dans les tableaux, aussi bien Marc Antoine que Jules César semblent fascinés par la situation inattendue de leur rencontre. Ils restent sûrement sous le charme de la grande beauté de la reine et de son environnement exotique, mais aucun indice ne nous laisse penser qu'ils se sentent totalement dépendants d'elle, sous l'emprise de sa fatalité féminine.

En revanche, il paraît intéressant de signaler que nous pouvons observer de nombreuses ressemblances entre les représentations artistiques et la création poétique inspirées par le moment de la mort de Cléopâtre. Tant les peintures académiques que le sonnet de Zawistowska, riche en expressions parnassiennes, nous apportent des visions pittoresques, colorées et vigoureuses. Il faut constater qu'elles sont beaucoup plus théâtrales que dramatiques, quant à la situation présentée. Cette approche est bien visible dans la phrase finale du sonnet: «Et le reptile a enlacé son buste de reine (...) d'une ceinture d'émeraudes, meurtrière. $»^{49}$

En ce qui concerne une autre grande femme fatale du XVIII ${ }^{e}$ siècle, Madame de Pompadour, Zawistowska a commis une erreur en la liant à la personne du Roi Soleil. Même en faisant abstraction de cette faute historique, un Louis XIV ou un Louis XV n'est pour la poétesse qu'un amant qui prend une leçon d'amour chez sa favorite. C'est la maitresse raffinée et fatale pour laquelle le roi est capable de jeter toute la France à ses pieds en « s'abîmant dans l'extase du désir " ${ }^{50}$.

Nous nous sommes permis de réserver pour la fin de ces quelques réflexions sur les femmes historiques le personnage d'Agrippine qui est devenu pour Zawistowska un exemple de femme-hybride. Sa cruauté et sa sensualité sont comparées au comportement d'une tigresse sauvage. ${ }^{51}$ C'est une figure bien connue dans la littérature européenne. Décrivant les formes zoomorphes - les femmes-félins - dans la culture moderne, Alain François cite aussi plusieurs œuvres littéraires et picturales du XIX ${ }^{\mathrm{e}}$ siècle, dans lesquelles il trouve des images particulièrement intéressantes de femmes. 
Dans la plupart de ces exemples, il ne s'agit pas encore tout à fait de figures zoomorphes, de femmes-hybrides, mais plutôt d'êtres ressemblant à des chattes. Toutefois, et ce fait mérite d'être souligné dans le contexte de notre réflexion, elles savent tenter et blesser les hommes. François accentue en particulier leurs aspects visuels et leur caractère ambivalent :

En littérature (...), généralement la femme-chatte n'est pas un monstre, mais une humaine «normale » qui se démarque par son apparence, son comportement, ses accessoires, voire ses animaux domestiques... Souvent elle est brune, répondant à un fantasme exotique de la bourgeoisie du XIX siècle. La fille du sud, nécessairement fougueuse, sauvage toujours, semble mieux répondre au modèle $\mathrm{du}$ chat, c'est la Carmen de Mérimée, la « Vieille Maîtresse »de Barbey d'Aurevilly ou la «Concha »de Pierre Louÿs. Elle doit être souple et hautaine, comme le chat, sensuelle évidement, et pouvoir blesser, soit moralement, soit physiquement. On pourrait parfaitement classer dans les femmes félines la quasi-intégralité des séductrices de roman, puisque le motif, par ses qualités spécifiques, vient se calquer sur celui de la brune malfaisante perpétuellement opposée à la blonde ingénue. ${ }^{52}$

Ces images ne sont pas vraiment éloignées de celles des personnages fantastiques que nous retrouvons aussi bien dans la poésie que dans la peinture de la Jeune Pologne. Elles appartiennent à un genre spécifique de «Fleurs du Mal» et de «Bestiaire d'Amour " ${ }^{53}$, mais correspondent bien aux nombreuses manifestations de la fin de siècle en Europe. Avant tout, il nous faut nommer les Chimères et les Harpies qui apparaissent sur les toiles du peintre polonais Jacek Malczewski. Ces monstres mythiques, fabuleux aux torses de femme, aux cheveux roux, aux griffes acérées et au regard séduisant sont présentés dans l'œuvre de Malczewski, dans des contextes divers. Les fantasmagories apparaissent en compagnie de petits bergers innocents (Le Petit berger à la Chimère, 1904) mais aussi d'artistes mûrs et éprouvés. Les hommes, malgré leur expérience de la vie, cèdent à la beauté de la Chimère qui symbolise ici un aspect sensuel de l'art (L'Artiste et la Chimère, 1906 ; Le moment de la création - La Harpie dans le sommeil, 1907).

Parmi les images fantastiques qui relient une silhouette féminine au monde animal, nous remarquons encore celles qui figurent sur les toiles d'Edward Okuń. Malheureusement, les œuvres originales représentant les hybrides ont disparu, mais nous connaissons leurs reproductions sur lesquelles nous pouvons distinguer: une femme-lézard (L'après-midi d'été. Le Lézard, avant 1906), une femme-léopard (Mademoiselle Léopard, vers 1906), une femme-paon (La Paonne, vers 1905 ; L'Enivrement de printemps, vers 1906). Tous ces personnages, beaux et sensuels, incarnent un instinct sexuel, une grande passion, les secrets et les mystères de la femme. Un critique anonyme écrivait en 1906 à propos de l'une de ces peintures :

La Paonne d'Edward Okuń est une personnification de la vanité propre à un certain type de femmes. (...) Le personnage qui se transforme en bas en un oiseau aux plumes de paon, est présenté sur le fond d'un paysage d'une extraordinaire qualité. Le peintre a rangé ces plumes avec une telle habileté qu'elles forment une sorte de robe qui traîne, brillante et riche. Un fléchissement de la tête de la femme et de son corps, un mouvement de ses mains - tout cela exprime le désir d'éveiller une admiration. ${ }^{54}$

41 Un deuxième type de personnages hétérogènes est celui de la femme qui se métamorphose en plante. Le motif est connu depuis l'Antiquité, surtout grâce aux Métamorphoses d'Ovide qui contiennent l'histoire de la transformation de Daphné en laurier. ${ }^{55}$ Nous retrouvons aussi ce mode de métamorphoses dans la littérature polonaise, par exemple dans l'œuvre de Jan Kochanowski et d'autres poètes de l'époque de la Renaissance et du Baroque $^{56}$, et puis dans la littérature et l'art fin de siècle aussi bien polonais 
qu'européens. Certaines œuvres choisies d'Odilon Redon et les visions hallucinatoires de Jean des Esseintes du roman de Huysmans se distinguent néanmoins des autres. Rappelons un de ses rêves maladifs, dans lequel apparaît une femme-fleur odieuse, un être sinistre et fatal qui sait détruire chaque homme. C'est l'incarnation de la Grande Vérole:

Sur le sol quelque chose remua qui devint une femme très pâle, nue, les jambes moulées dans des bas de soie verts.

Il la contempla curieusement; semblables à des crins crespelés par des fers trop chauds, ses cheveux frisaient, en se cassant du bout; des urnes de Népenthès pendaient à ses oreilles; des tons de veau cuit brillaient dans ses narines entr'ouvertes. Les yeux pâmés, elle l'appela tout bas.

Il n'eut pas le temps de répondre, car déjà la femme changeait; des couleurs flamboyantes passaient dans ses prunelles; ses lèvres se teignaient du rouge furieux des Anthurium (...)..$^{57}$

Dans la littérature polonaise, seules les représentations des femmes imaginées par Przybyszewski dans ses poèmes en prose auraient pu rivaliser avec celles de Huysmans sur le plan de l'imagination poétique. Pourtant, elles étaient loin d'être les seules à figurer des personnages hétérogènes dans la prose et dans la poésie de la Jeune Pologne. Nous relevons les métaphores florales et animales liées à l'image féminine notamment dans le roman de Wacław Berent Le Blé d'hiver ${ }^{58}$, mais les descriptions les plus saisissantes abondent dans les poèmes de Tetmajer, d'Ostrowska, de Zawistowska ou de Jerzy Żuławski. C'est là que nous rencontrons une maîtresse-fleur ${ }^{59}$, une femmelionne ${ }^{60}$, une femme-cygne ${ }^{61}$, une femme-sphinge $e^{62}$, une fille-Chimère ${ }^{63}$ ou une naïadepieuvre attendant son rêve - le petit bateau qui n'arrive pas : «Et j'attends des siècles infinis / Ton passage solitaire et lointain / Étendue sur un cercle d'immensité / Avec le désir des mains dans l'attente ${ }^{64}$.

Cette double nature de la femme, si accentuée à l'époque, s'exprime dans tous ces exemples par des formes variées et riches qui font fusionner deux êtres: un être humain et un animal ou un végétal. Toute la fatalité de la femme se cache dans cette deuxième nature, sous le masque de son deuxième visage.

\section{Conclusions}

Passons aux conclusions de nos réflexions sur les types d'images de la femme fatale dans la peinture et la poésie de la Jeune Pologne en regard des créations européennes. Premièrement, il faut constater une ressemblance frappante dans les motifs iconographiques des œuvres artistiques et littéraires de l'époque. L'imagination des peintres et des écrivains de toute l'Europe, y compris de la Pologne, puisait dans des sources identiques. Évidemment, elle ne pouvait pas être et elle n'était pas la même dans chaque pays et dans chaque nation, en raison des différences historiques, sociales ou culturelles. Mais, malgré toutes ces distinctions, les peintres et écrivains aussi bien français, belges, allemands que polonais, cherchaient des moyens artistiques et littéraires communs pour exprimer tout ce qui semblait indescriptible et imperceptible. Parmi les idées récurrentes, la nature de la féminité était l'une des plus intrigantes, excitant partout la curiosité. L'image de la femme, après avoir changé plusieurs fois au cours du XIX ${ }^{e}$ siècle, est devenue, au seuil de la modernité ambivalente, mystérieuse et fatale - aussi bien pour les artistes et les écrivains de l'Europe de l'ouest que pour ceux de Pologne. Après avoir analysé plusieurs œuvres, nous devons constater que toutes les 
incarnations de la femme fatale (que ce soit celle «à la polonaise » ou celle provenant de l'Europe de l'ouest) sont pourvues des mêmes traits principaux: la beauté, la sensualité, la volupté ou encore un grand mystère.

Pour conclure, nous nous permettrons d'insérer une citation datant des années 1860 afin de rappeler la thèse de Praz évoquée au début de notre article, selon laquelle, de tous temps, aussi bien au temps de Baudelaire, des Parnassiens, des grandes écoles réalistes ou naturalistes, la femme a été perçue comme un être incompréhensible et « impénétrable ». Cette idée est parfaitement rendue par Victor Hugo dans un de ses romans les plus célèbres :

Vous regardez une étoile pour deux motifs, parce qu'elle est lumineuse et parce qu'elle est impénétrable. Vous avez auprès de vous un plus doux rayonnement et un plus grand mystère, la femme. ${ }^{65}$

\section{NOTES}

1. Praz M., Zmysły, śmierć $i$ diabet $w$ literaturze romantycznej (La chair, la mort et le diable dans la littérature du XIX ${ }^{\mathrm{e}}$ siècle), traduction de Krzysztof Żaboklicki, PIW, Varsovie, 1974, p. 167.

2. Ibid., pp. 169-170.

3. Bajda J., « Kobieta młodopolska " (La femme de la Jeune Pologne), in : Bajda Justyna et alii, Epoki literackie. Wielki leksykon literatury polskiej (Les Époques littéraires. Le Grand Lexique de la Littérature Polonaise), Wydawnictwo DolnoślĄskie, Wrocław, 2005, pp. 663-664.

4. Gutowski W., Nagie dusze i maski. O młodopolskich mitach miłości (Les âmes nues et les masques. Des mythes d'amour dans l'époque de la Jeune Pologne), Wydawnictwo Literackie, Cracovie, 1997, pp. 3 \& 21. Toutes les traductions en français des citations en polonais sont de l'auteure du présent article.

5. Sur la peinture de salon en Pologne, voir: Poprzęcka M., Polskie malarstwo salonowe (La peinture de salon en Pologne), Wydawnictwa Artystyczne i Filmowe, Varsovie, 1991, 112 p. Dans ce contexte, nous pouvons citer le peintre Franciszek Żmurko, un des portraitistes polonais les plus populaires, et ses œuvres, dont : Kobieta $z$ wachlarzem (La femme à l'éventail, 1884) et Portret kobiety z wachlarzem i papierosem (Portrait d'une femme à l'éventail et à la cigarette,1891).

6. Par exemple: Portret pani Żukotyńskiej. Sfinks (Portrait de Madame Żukotyńska. Le Sphinx, 1928) ou Portret kobiecy (Portrait de femme, 1929); etc.

7. Ripa C., Iconologia overo Descrittione dell'Imagini Universali cavate dall'Antichità et da altri luoghi (Iconologie, ou Explication nouvelle de plusieurs images, emblèmes et autres figures hiéroglyphiques des vertus, des vices, des arts, des sciences et des passions humaines), Rome, 1593 (l'œuvre a été traduite en français par Jean Baudoin en 1643).

8. Par exemple : Portret kobiety (Portrait de femme, 1898 et 1907); Rudowłosa (Une rousse, 1899) et autres.

9. La peinture citée est très représentative mais peu connue.

10. Charazińska E., «Ćma nocna » (Papillon de nuit), in : Koniec wieku. Sztuka polskiego modernizmu 1890-1914 (La fin de siècle. L'art du modernisme polonais 1890-1914), sous la direction de Charazińska E. \& Kossowski Ł., éd. Muzeum Narodowe w Warszawie, Varsovie, 1996, p. 210.

11. Wierzchowska W., "Szał uniesień - obraz Władysława Podkowińskiego» (La Folie - un tableau de Władyslaw Podkowiński), in : Sztuka i krytyka. Materiały do studiów i dyskusji z zakresu 
teorii $i$ historii sztuki, krytyki artystycznej oraz badań nad sztukA, t. VII, cahier 3-4, éd. Państwowy Instytut Sztuki, Varsovie, 1957, pp. 88-103.

12. Kisielewski J. A., «W sieci (Dans la toile), in: Dramaty (Drames), sous la direction de Taborski R., Wydawnictwo Ossolineum, Wrocław, 1969, pp. 40-42.

13. Les autres poèmes d'après le tableau de Władysław Podkowiński ont été réunis et analysés dans le livre: Bajda J., "Poeci - to sA słów malarze... » Typy relacji miĘdzy słowem a obrazem w ksiĄżkach poetyckich okresu Młodej Polski («Les poètes sont les peintres des mots... » Les types de relations entre le mot et l'image dans les livres de poésie de l'époque de la Jeune Pologne), Wydawnictwo Uniwersyteckie, Wrocław, 2012, chapitre: Poetyckie interpretacje "Szału» Władysława Podkowińskiego (Les interprétations poétiques de «La Folie» de Władysław Podkowiński), pp. 107-115.

14. Gordziałkowska Z., Szał. Obraz Podkowińskiego (La Folie. Le tableau de Podkowiński), in : W samotności (Dans la solitude), éd. Gebethner i Spółka, Cracovie, 1910, p. 24.

15. Idem.

16. Idem.

17. Maria Komornicka est tombée dans l'apathie de la fin du siècle, puis a sombré dans une maladie nerveuse. Elle a brûlé ses vêtements de femme, a remplacé son nom par celui de Piotr Włast et a demandé d'être traitée comme un homme.

18. Komornicka M., « Pod wpływem“Szału” Podkowińskiego » (Sous l’influence de «La Folie » de Podkowiński), in: Nałkowski W., Komornicka M. \& Jellenta C., Forpoczty (Les Avant-postes), éd. par les auteurs, Lviv, 1895,p. 200.

19. Przerwa-Tetmajer K., «Obraz Podkowińskiego » (Le tableau de Podkowiński), in: Kurier Codzienny, $\mathrm{n}^{\circ} 77,1894$, p. 2.

20. Przerwa-Tetmajer K., «Ja, kiedy usta... » (Moi, quand j'approche ma bouche...), in : Poezje. Wydanie zbiorowe (Poésies. Édition complète), vol. I., éd. Instytut Wydawniczy, Biblioteka Polska, Varsovie, 1924, p. 87 ; « Lubię, kiedy kobieta... » (J'aime, quand une femme...), in : Ibid., p. 203.

21. Przerwa-Tetmajer K., «Leda »(Léda), in : Poezje, op. cit., p. 196.Nous ne pouvons être sûrs que Kazimierz Przerwa-Tetmajer sût que l'œuvre de Michel-Ange avait disparu des siècles auparavant et qu'il décrivît un tableau de Peter Paul Rubens, fait aux alentours de 1600 d'après une peinture de Michel-Ange.

22. Przerwa-Tetmajer K., « Danae Tycjana »(Danaé du Titien), in : Poezje, op. cit.,vol. II, p. 41.

23. Przerwa-Tetmajer K., «Obraz Podkowińskiego », art. cit., p. 2.

24. Sur les femmes dans la vie de Stanisław Przybyszewski : Kolińska K., Stachu, jego kobiety i jego dzieci (Stachu, ses femmes et ses enfants), éd. KsiĄżka i Wiedza, Varsovie, 1994, 224 p.

25. Sur la vie de Dagny Przybyszewska: Sawicka A., Dagny Juel Przybyszewska. Fakty i legendy (Dagny Juel Przybyszewska. Faits et légendes), Wydawnictwo słowo/obraz terytoria, Gdańsk, 2006, $438 \mathrm{p}$.

26. Przybyszewski St., Androgyne, in : Poematy prozA (Poèmes en prose), Podraza-Kwiatkowska M. \& Kwiatkowski J. (éd.), Wydawnictwo Literackie, Cracovie, 2003, p. 407. Le dessin de Félicien Rops d'après lequel Przybyszewski a inventé la scène célèbre dans son poème en prose est La Tentation de saint Antoine.

27. Przybyszewski St., Na drogach duszy (Sur les chemins de l’âme), éd. L. Zwoliński i Spółka, Cracovie, 1902, pp. 26-27.

28. Lange A., «Lilith», in : Rozmyślania (Méditations), éd. Wacław Wiediger, Cracovie, 1906, p. 113.

29. Lady Lilith de Dante Gabriel Rossetti (1869).

30. Lilith de John Collier (1892).

31. Konopnicka M., « Z powodu wystawy secesjonistów w Wiedniu » (À cause de l'exposition des artistes de la Sécession à Vienne), in : Tygodnik Ilustrowany, nº 3, 1895, p. 207.

32. L'histoire de Judith : Ancien Testament, Livre de Judith. 
33. L'histoire de l'exécution de saint Jean-Baptiste : Nouveau Testament, Évangile selon saint Marc (VI : 14-29), Évangile selon saint Matthieu (XIV : 1-12). La Bible ne donne pas de détails en ce qui concerne Salomé, la fille d'Hérodiade - son prénom n'est d'ailleurs pas présent dans les textes bibliques. Nous le connaissons grâce aux informations données par Flavius Josèphe ( $\mathrm{I}^{\mathrm{er}}$ siècle après Jésus-Christ) dans son œuvre principale L'Histoire ancienne d'Israël.

34. Huysmans J.-K., À rebours, éd. Pour les cent bibliophiles, Paris, 1903, pp. 56-62.

35. Rabska Z., "Salome. Moreau ", in : W płonAccym lesie. Poezje (Dans une forêt incendiée. Les poésies), éd. Gebethner i Wolff, Varsovie, 1918, p. 88.

36. Wilde O., Salomé : Drame en un acte, Librairie de l'art Indépendant, Paris, 1893, pp. 80-81.

37. Ibid., p. 84.

38. Gutowski W., op. cit., p. 36.

39. Kasprowicz J., "Salome »(Salomé), in: GinĄcemu światu (Pour le monde mourant), éd. Towarzystwo Wydawnicze, Lviv, 1902, pp. 23-44. Les autres œuvres: Chrystus. Poemat społeczno-religijny (Le Christ. Poème socio-religieux), éd. KsiĄżka, Cracovie, 1904 ; Uczta Herodiady. Poemat dramatyczny $w$ trzech aktach (Le Banquet d'Hérodiade. Poème dramatique en trois actes), éd. KsiĘgarnia H. Altenberga, Lviv, 1905.

40. Praz M., op. cit., pp. 169-170.

41. Zawistowska K., « Herodiada »(Hérodiade), in : Poezje (Poésies), éd. KsiĘgarnia H. Altenberga, Lviv, 1903, p. 52.

42. Daniłowski G., Maria Magdalena (Marie Madeleine), éd. Ksį̨garnia J. Czerneckiego, Varsovie, 1912.

43. Trześniowski D., Modernistyczny portret grzesznic Nowego Testamentu (Le portrait moderniste des pécheresses du Nouveau Testament), in : Kobiety $w$ literaturze $i$ kulturze (Les femmes dans la littérature et dans la culture), sous la direction de Mazurek D , Wydawnictwo Uniwersytetu Marii Curie-Skłodowskiej, Lublin, 2004, p. 149.

44. Ostrowska Br., Eloe i Magdalena (Eloé et Madeleine), in : Aniołom dźwiĘku (Aux Anges du son), éd. Gebethner i Wolff, Varsovie, 1913, pp. 161-176.

45. Ostrowska Br., Magdaleno, ciszo polna... (Madeleine, silence de champ !...), in : Pierścień życia (L’anneau de la vie), éd. Kasa Przezorności i Pomocy Warszawskich Pomocników Księgarskich, Varsovie, 1911, p. 11.

46. Trześniowski D., op. cit., p. 154.

47. Zawistowska K., « Kleopatra »(Cléopâtre), in : Poezje, op. cit., p. 51.

48. Par exemple: Sir Lawrence Alma-Tadema, Jean-Léon Gérôme, John William Waterhouse, Frederick Arthur Bridgman, Alexandre Cabanel.

49. Zawistowska K., « Kleopatra », op. cit.

50. Zawistowska K., « Pompadour », in : Poezje, op. cit., p. 53.

51. Zawistowska K., « Agrypina »(Agrippine), in : Ibid., p. 50.

52. François A., «La femme-félin, figure zoomorphe transculturelle ", in : Culture visuelle (Média social d'enseignement et de recherche) [En ligne]. URL : http://culturevisuelle.org/detresse/ archives/20 (document consulté le 27 décembre 2012).

53. Gutowski W., op. cit., chapitre : «Kwiaty zła i miłosne bestiarium » (Les Fleurs du mal et le bestiaire d'amour), pp. 44-57.

54. Citation d'après : Biernacka M., Literatura - Symbol - Natura (La Littérature - Le Symbole - La Nature), Instytut Sztuki PAN, Varsovie, 2004, p. 81.

55. Ovide, Przemiany (Les Métamorphoses), traduction de Kiciński B., sous la direction de Krókowski J., éd. Ossolineum, Wrocław, 1953, pp. 21-26 (I, 455-576).

56. Par exemple : Kochanowski J., « Pieśń świętojańska o Sobótce » (Le chant de la Saint-Jean), in : Pieśni (Les Chants), sous la direction de Szczerbicka-Ślęk L., éd. Ossolineum, Wrocław, 1997, pp. 90-112 ; Zimorowic Sz., « Lenerula », in: Roksolanki, sous la direction de Ślękowa L., éd. Zakład 
Narodowy im. Ossolińskich, Wrocław, 1983, pp. 37-39; Twardowski S., «Dafnis drzewem bobkowym »(La Transformation de Daphné en laurier), in: Dafnis drzewem bobkowym,sous la direction de Okoń J., éd. Ossolineum, Wrocław, 1976, pp. 1-191.

57. Huysmans J.-K., op. cit., p. 99-100.

58. Berent W., Ozimina (Le Blé d'hiver), éd. Ossolineum, Wrocław, 1974.

59. Przerwa-Tetmajer K., « Kwiat symboliczny »(La Fleur symbolique), in : Poezje, op. cit., vol. I., p. 182.

60. Zawistowska K., « Lwica »(La Lionne), in : Poezje, op. cit., p. 49.

61. Wolski W., «Kobieta-łabędź »(La Femme-Cygne), in: Powieść tajemna (Le roman secret), éd. Gebethner i Sp., Cracovie, 1908, p. 83.

62. Wolski W., "Loo Toroo ", in : Powieść tajemna. Poezje. Seria druga (Le roman secret. Poésies. Deuxième Série), éd. Gebethner i Spółka, Cracovie, 1908, p. 6. Le poème de Wacław Wolski, placé dans le contexte de la peinture de Rops et du livre de Jules Barbey d'Aurevilly Les Diaboliques, a été decrit par Maria Podraza-Kwiatkowska dans le livre Symbolizm i symbolika w poezji Młodej Polski (Le Symbolisme et la Symbolique dans la poésie de la Jeune Pologne), éd. Universitas, Cracovie, 1994, pp. 133-135.

63. Rydel L., «Chimera »(La Chimère), in : Poezje (Poésies), éd. Leon Idzikowski, Cracovie, 1909, pp. 37-41.

64. Ostrowska Br., « Ośmiornica »(La Pieuvre), in : Biała godzina. Wybór poezji (L'Heure blanche. Choix de poésie), PIW, Varsovie, 1982, p. 84.

65. Hugo V., Les Misérables, éd. Émile Testard, Paris, 1891.

\section{RÉSUMÉS}

Dans le présent article, je me concentre sur trois représentations de la femme fatale «à la polonaise ", qui correspondent à des figures célèbres de la littérature et de l'art européens de la fin $\mathrm{du} \mathrm{xix}^{\mathrm{e}}$ siècle. Tout d'abord, je présente les figures de femmes fatales contemporaines (appartenant entièrement à la réalité de l'époque de la Jeune Pologne). Puis, je propose de distinguer un ensemble de figures bibliques. Ces héroïnes apparaissent aussi bien dans la peinture et la littérature de la Jeune Pologne que dans celles de toute la Jeune Europe. Dans l'art polonais, je distingue plusieurs personnages de femmes de l'Ancien et du Nouveau Testament (Lilith, Ève, Judith, Hérodiade, Salomé et Marie Madeleine). Dans un dernier temps, je m'intéresse à des types de femmes plus rares dans l'art et dans la littérature de la Jeune Pologne. Il s'agit des personnages historiques, mythiques et surtout fantastiques (la femme-hybride). L'objectif principal de l'article est de présenter aux lecteurs francophones différentes images des femmes fatales issues de la création littéraire et artistique de la Jeune Pologne, en montrant les caractéristiques qu'elles partagent avec les créations artistiques européennes. Les exemples sont tirés d'une part d'œuvres poétiques et romanesques et d'autre part de productions artistiques. 
INDEX

Index chronologique : Belle époque, fin de siècle, Jeune Pologne

Index géographique : Cracovie, Paris, Pologne

Mots-clés : femme fatale, femme-cygne, femme-hybride, femme-lionne, femme-sphinge, fille-

Chimère, maîtresse-fleur, peinture, personnage hétérogène, poésie

\section{AUTEURS}

JUSTYNA BAJDA

Docteur habilité, maître de conférences de la Faculté des Lettres, Institut de Philologie Polonaise, Université de Wrocław (Pologne) 